\title{
DESARROLLO DE UN PROGRAMA DE EJERCICIO TERAPÉUTICO COLABORATIVO, CON PERSPECTIVA DE GÉNERO, EN EL GRADO DE FISIOTERAPIA
}

\author{
Naia Entonado, Zeltia'; Bello Rodríguez, Olalla²; Carballo Costa, Lidiaª \\ ${ }^{1}$ Universidade da Coruña, Facultade de Fisioterapia, id0000-0001-7878-4018 \\ ${ }^{2}$ Universidade da Coruña, Facultade de Fisioterapia, id0000-0002-7846-3123 \\ ${ }^{3}$ Universidade da Coruña, Facultade de Fisioterapia, id0000-0003-3674-2789
}

\section{RESUMO}

El movimiento, como agente físico aplicado con un fin terapéutico, es la base de la Cinesiterapia. Se constituye como un pilar fundamental dentro del curriculum del grado de Fisioterapia. Dentro de la asignatura "Cinesiterapia: bases del ejercicio terapéutico", se enseña al alumnado a realizar programas de ejercicio terapéutico basados en la evidencia científica disponible, de forma grupal e individual. Con el objetivo de motivar al alumnado, y trabajar una perspectiva de género, se propone como actividad el diseño de programas de ejercicio terapéutico de forma grupal y colaborativa. El objetivo de esta experiencia es diseñar por grupos un programa de ejercicio terapéutico y favorecer un proceso de reflexión sobre las diferencias de género en la respuesta cardiovascular. Esta dinámica mejora la motivación de los estudiantes y su participación en el proceso de aprendizaje.

PALABRAS CLAVE Fisioterapia, ejercicio terapéutico, kinesiterapia, resistencia, mujeres 


\section{CITA RECOMENDADA:}

Naia Entonado, Zeltia; Bello Rodríguez, Olalla; Carballo Costa, Lidia (2021): Desarrollo de un programa de ejercicio terapéutico colaborativo, con perspectiva de género, en el grado de Fisioterapia. En García Naya, J.A. (ed.) (2021). Contextos universitarios transformadores: a nova normalidade académica. Leccións aprendidas e retos de futuro. V Xornadas de Innovación Docente. Cufie. Universidade da Coruña. A Coruña (pág. 261-265).

DOI capítulo: https://doi.org/10.17979/spudc.9788497498180.261

DOl libro: https://doi.org/10.17979/spudc.9788497498180

\section{ABSTRACT}

Movement, as a physical agent applied with a therapeutic goal, is the basis of Kinesiotherapy. It is a fundamental pillar of the curriculum of Physiotherapy degree program. Within the subject "Kinesiotherapy: basis of therapeutic exercise", the students learn how to design programs of therapeutic exercise based on the available scientific evidence. The design of therapeutic exercise programs is proposed, in order to motivate the students, and working on a gender perspective. It's stablished as a grupal and collaborative activity. The outcome of this experience is designing in groups a therapeutic exercise program and a reflexion proccess about gender differences in the cardiovascular response. This dynamic implies the reflexion about the different cardiovascular responses and adaptations of women to exercise, and also how to desing justified activities for them. This activity improves motivation and participation of the students.

KEY WORDS: Physical therapy, physical therapy modalities, exercise therapy, kinesitherapy (no es un término mesh), women 


\section{INTRODUCCIÓN}

La cinesiterapia se define como el uso del movimiento, un agente físico, con un fin terapéutico. Se constituye como un pilar fundamental dentro del curriculum del grado de Fisioterapia.

El ejercicio terapéutico es la realización sistematizada y planificada de movimientos corporales, posturas y actividades que tienen por objetivo remediar o prevenir alteraciones, mejorar la función física, prevenir o reducir factores de riesgo relacionados con la salud, optimizar el estado de salud, la condición física 0 la sensación de bienestar ${ }^{1}$. Dentro de los contenidos de la asignatura "Cinesiterapia: bases del ejercicio terapéutico", se encuentra el desarrollo de las capacidades físicas básicas, y el diseño de programas de ejercicio terapéutico. La resistencia es una capacidad física básica, que permite resistir a la fatiga durante un mayor tiempo, o realizar un esfuerzo de forma eficaz durante el mayor tiempo posible ${ }^{2,3}$. Para favorecer la implicación del alumnado así como la participación e interacción entre ellos, se desarrolla esta dinámica grupal y colaborativa. Contribuyó a aumentar el proceso de debate, reflexión y diseño de actividades en grupo y de manera colabotariva.

\section{DESCRIPCIÓN DE LA EXPERIENCIA}

Se propusieron seis casos clínicos distintos, para que el alumnado elaborase, de forma colaborativa, un programa de ejercicio terapéutico orientado a la capacidad física de la resistencia. En caso de que el perfil del paciente fuese una mujer, debían reflexionar sobre las características diferenciadoras en la respuesta cardiovascular, y elaborar su programa de ejercicio terapéutico en función de ellas. Posteriormente, se dividió al alumnado en 6 subgrupos distintos, que se corresponden con sus grupos de docencia práctica.

Antes de la realización de los mismos, el profesorado explicó las características de la Resistencia, como capacidad física; se grabó un vídeo, que estuvo a disposición del alumnado 3 días antes de la clase síncrona. En la clase síncrona, se desglosaron las características del cálculo de la intensidad de la resistencia. Se realizó a través de la visualización y explicación de una presentación síncrona, y de la aplicación "Whiteboard". Dicha información quedó a 
disposición del alumnado. A continuación, se describieron las características diferenciadoras de las respuestas cardiovasculares de las mujeres al ejercicio físico. En la Tabla 1 se reflejan las más importantes ${ }^{3-5}$ :

\begin{tabular}{|c|c|}
\hline Cardiovascular & $\begin{array}{l}\text { - } \quad \text { <S: < tamaño corazón, > FC, para un trabajo submáximo, } \\
\text { misma I esfuerzo (hombres) } \\
\text { - } \quad<\text { volumen sanguíneo, Hb } \\
\text { - } \quad>\text { grasa }\end{array}$ \\
\hline Respiratorio & $\begin{array}{ll}\text { - } & <\text { volumen pulmonar } \\
\text { - } & <\text { flujo espiratorio máximo } \\
\text { - } & >\text { FR fase lútea* }\end{array}$ \\
\hline Hormonal & $\begin{array}{ll}\text { - } & \text { Fase lútea - fase folicular } \\
\text { - } & \text { Diferencias en parámetros: ciclos menstruales regulares }\end{array}$ \\
\hline
\end{tabular}

Tabla 1: Diferencias de género en las respuestas y adaptaciones al ejercicio

\section{RESULTADOS}

Se diseñan 6 programas de ejercicio terapéutico de forma grupal. Un alumno 0 alumna de cada grupo se encarga de remitirlo al profesorado de la asignatura, cuando se obtenga una versión de consenso. De los 6 programas de ejercicio terapéutico, 3 están basados en perfiles de mujeres. Para valorar la experiencia, se diseñó un cuestionario para identificar la opinión del alumnado sobre las experiencias de innovación docente realizadas en la materia. Se elaboró una pregunta sobre el desarrollo de este programa de ejercicio, en la que se solicitaba al alumnado su grado de acuerdo o desacuerdo con la siguiente afirmación: "Identificar las respuestas y adaptaciones cardiovasculares al ejercicio en mujeres, me ha resultado útil para comprender las posibles diferencias de género". El alumnado debía valorar, en una escala numérica, su grado de acuerdo 0 desacuerdo; la puntuación 1 significaba "estoy completamente en desacuerdo", y la puntuación 7 significaba "Estoy completamente de acuerdo". De un total de 22 respuestas, el valor mínimo fue 3, el valor máximo 7, y el valor media 5,2 . 


\section{CONCLUSIONES}

El desarrollo de un programa de ejercicio terapéutico basado en la capacidad física de la resistencia con perspectiva de género, resulta novedoso para el alumnado. Favorece el debate sobre las diferencias en la respuesta cardiovascular de las mujeres frente a los hombres, la integración de los conocimientos trabajados en clase asíncrona y síncrona, así como el intercambio de opiniones entre el alumnado. Con esta experiencia, aumentó la interacción del alumnado en docencia virtual síncrona, a través del desarrollo de una tarea grupal y se introdujo la reflexión sobre la perspectiva de género en el diseño de programas de ejercicio terapéutico. Para futuras experiencias, podría fomentarse el autoaprendizaje con materiales de ampliación y apoyo, así como desarrollar este tipo de programas de forma más frecuente.

\section{REFERENCIAS}

Kisner C C LA. (2010). Ejercicio terapéutico: Fundamentos y técnicas. Paidotribo.

Bushnell MC, Ceko M, Low LA. (2013). Cognitive and emotional control of pain and its disruption in chronic pain. Nat Rev Neurosci, 14(7):502-511.

López Chinarro, J, Fernández Vaquero A. (2008). Fisiología del ejercicio. Madrid, 3a Ed.: Editorial Medica Panamericana.

Sheel AW. (2016). Sex differences in the physiology of exercise: an integrative perspective. Exp Physiol, 101(2):211-212.

Day DS. (2008). Exercise physiologists talk about sex differences. Med Sci Sports Exerc, 40(4):646-647 
